\title{
FRACTIONAL BIORTHOGONAL PARTNERS AND APPLICATION IN SIGNAL INTERPOLATION
}

\author{
Bojan Vrcelj and P. P. Vaidyanathan \\ Dept. of Electrical Engr. 136-93, Caltech, Pasadena, CA 91125, USA \\ E-mail: bojan@systems.caltech.edu,ppvnath@systems.caltech.edu
}

\begin{abstract}
The concept of biorthogonal partners has been introduced recently by the authors. The work presented in this paper is an extension of some of these results to the case where the upsampling and downsampling ratios are not integers but rational numbers. Hence the name fractional biorthogonal partners. The conditions for the existence of stable and of FIR fractional biorthogonal partners are derived. This result gives rise to an all-FIR spline interpolation technique with the minimum amount of required oversampling. This technique is illustrated by an interpolation example.
\end{abstract}

\section{INTRODUCTION}

The concept of biorthogonal partners has been introduced recently by the authors in both the scalar [5] and the vector case [8]. Digital filters $H(z)$ and $F(z)$ are called biorthogonal partners of each other with respect to an integer $M$ if their cascade $H(z) F(z)$ obeys the Nyquist $(M)$ property. There is a strong connection between this definition and discrete signals oversampled by the integer amount $M$. In this paper we present an extension of the same reasoning to signals oversampled by fractional amounts. This gives rise to the definition of fractional biorthogonal partners (FBPs in the following).

We first provide a motivation for the study of FBPs and give their formal definition. Next we show a way to construct fractional biorthogonal partners. This discussion leads to deriving the conditions for the existence of FIR FBPs and of stable FBPs. After that we show one of the applications of these results, namely the cubic spline interpolation. We show that it is possible to interpolate a slightly oversampled image using exclusively FIR filtering. Some of the other applications of FBPs, including the use in fractionally spaced equalizers (FSEs) are explored in [7].

\subsection{Notations}

If not stated otherwise, all notations are as in [3]. We use the encircled symbol $\downarrow M$ to denote the decimation operation (turns $x(n)$ into $x(M n))$. The expanded version of $x(n)$

$$
\begin{cases}x(n / M) & \text { for } n=\text { mul of } M, \\ 0 & \text { otherwise }\end{cases}
$$

is similarly obtained as a result of the expander operation which is denoted by the encircled symbol $\uparrow M$.

Work supported in part by the ONR grant N00014-99-1-1002, USA

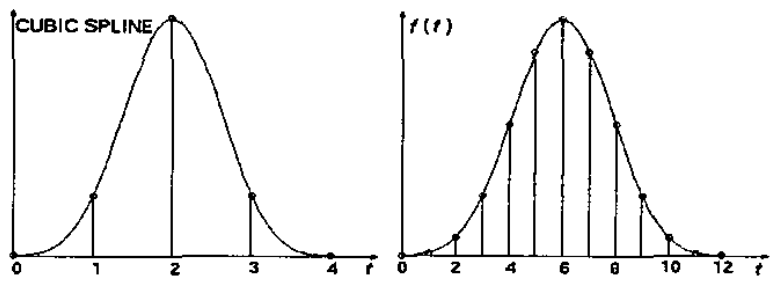

Fig. 1. Example of a generating function $\phi(t)$ (cubic spline) and its three times "stretched" version $f(t)$.

\section{FRACTIONAL BIORTHOGONAL PARTNERS}

Biorthogonal partners as originally introduced in [5] arise in many different contexts. One of them is the reconstruction of continuous time signals admitting the model

$$
x(t)=\sum_{k=-\infty}^{\infty} c(k) \phi(t-k) .
$$

Suppose we are given the discrete time signal $y(n)$ that is obtained by sampling $x(t)$ from (1) at the rate $L / M$, i.e. $y(n)=$ $x(n M / L)$. For obvious reasons we will assume that $M$ and $L$ are co-prime. We shall see later that for the purpose of this paper $L>M$ is required as well, although in principle it is not necessary. Now, we can say that $y(n)$ is obtained by oversampling $x(t)$ with respect to the usual integral sampling strategy by a factor of $L / M$. Then we have

$$
\begin{aligned}
y(n) & =x\left(\frac{M}{L} n\right)=\sum_{k=-\infty}^{\infty} c(k) \phi\left(\frac{M}{L} n-k\right) \\
& =\sum_{k=-\infty}^{\infty} c(k) f(M n-k L),
\end{aligned}
$$

where $f(t) \triangleq \phi(t / L)$ is the generating function "stretched" by a

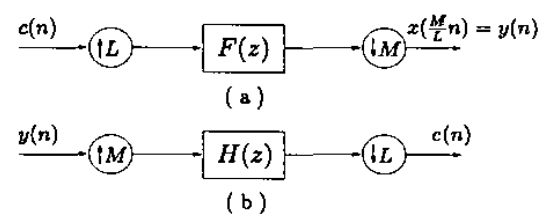

Fig. 2. (a) Signal model. (b) Scheme for reconstruction. 
(a)
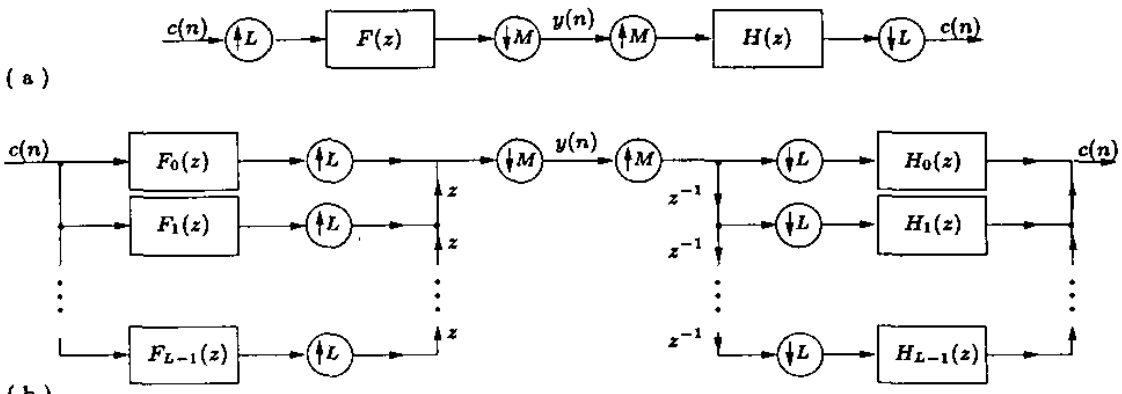

Fig. 3. (a)-(b) Equivalent presentations of fractional biorthogonal partners.

factor of $L$. This is shown in Fig. 1 for the case where $\phi(t)$ is a cubic spline [1] and $L=3$. The signal $y(n)$ from (2) can thus be obtained as shown in Fig. 2(a). The problem of signal reconstruction can be considered as that of finding the driving sequence $c(n)$ given the discrete signal $y(n)$. Obviously, if the sequence $c(n)$ is known, reconstruction of $x(t)$ for any given $t$ follows from (1).

We look for the solution of the form shown in Fig. 2(b). We will show that under some mild assumptions this solution [i.e. filter $H(z)$ ] exists. Further, we establish the conditions under which for an FIR filter $F(z)$ the corresponding reconstruction filter $H(z)$ can be FIR as well.

\subsection{Definition}

The preceding discussion leads naturally to the definition of fractional biorthogonal partners.

Definition. Transfer function $H(z)$ is said to be a right fractional biorthogonal partner (RFBP) of $F(z)$ with respect to the fraction $L / M$ if the system shown in Fig. 3(a) is identity. Under these conditions $F(z)$ is also said to be a left fractional biorthogonal partner (LFBP) of $H(z)$ with respect to $L / M$.

This definition includes the notion of (integral) biorthogonal partners [5] as a special case when $M=1$. Note that the system in Fig. 3(a) becomes linear time invariant (LTI) in the special case $M=1$, while in general is not. Also, note that (as opposed to the $M=1$ case) we need to distinguish between left and right FBPs. However, the results that hold for RFBPs can be easily modified to accommodate LFBPs, and therefore we only focus on RFBPs in the following. If the fraction $L / M$ is changed, the two filters may not remain fractional biorthogonal partners, but we will avoid mentioning this factor whenever no confusion is anticipated.

Now, returning to the previous discussion we see that the reconstruction of $x(t)$ given by the model (1) from its samples $y(n)$ obtained at rate $L / M$ is possible if $F(z)$ has a stable RFBP $H(z)$. It is possible to perform an FIR reconstruction if there exists an FIR RFBP. In the following we describe a way of constructing fractional biorthogonal partners. As a result we will have conditions for the existence of an FIR or just stable FBPs.

\subsection{Existence and construction of FBPs}

Consider the system in Fig. 3(a). Write the filters $F(z)$ and $H(z)$ in terms of their Type-2 and Type-1 polyphase components [3]

$$
F(z)=\sum_{k=0}^{L-1} F_{k}\left(z^{L}\right) z^{k}, \text { and } H(z)=\sum_{k=0}^{L-1} H_{k}\left(z^{L}\right) z^{-k} .
$$

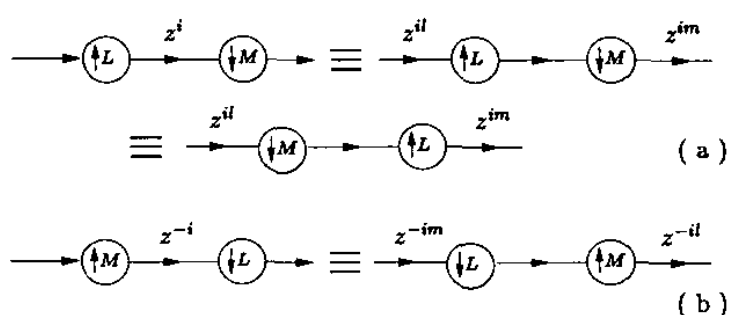

Fig. 4. (a)-(b) Some multirate identities.

Then we can redraw this system as shown in Fig. 3(b). Now, consider the left-hand side of Fig. 3(b) and focus on the system between the output of the $i$ th filter $F_{i}(z)$ and $y(n)$. This is given by a cascade of an expander by $L$, advance operator $z^{i}$ and a decimator by $M$. Since we assumed $M$ and $L$ are co-prime, there exist integers $m$ and $l$ such that

$$
l L+m M=1 .
$$

In fact, the unique solution for the smallest $m$ and $l$ can be obtained by the Euclid's algorithm. Writing the delay $z^{i}=z^{i l L} \cdot z^{i m M}$, we can easily prove the multirate identity depicted in Fig. 4(a). Similarly, we can show that the system between $y(n)$ and the input to $H_{i}(z)$ can be equivalently redrawn as in Fig. 4(b).

Therefore, substituting those changes in Fig. 3(b) we obtain the equivalent structure shown in Fig. 5(a). Let us now define

$$
P_{k}(z) \triangleq z^{k l} F_{k}(z), \text { and } Q_{k}(z) \triangleq z^{-k l} H_{k}(z),
$$

for $0 \leq k \leq L-1$. Since $L$ and $M$ are co-prime, it follows that $L$ and $m$ are co-prime as well. Under these circumstances it can be shown that the $L \times L$ system shown in Fig. 5(a) within the dashed box is the identity. Thus, the whole structure can be redrawn as in Fig. 5(b). It is important to notice here that the original filters $F(z)$ and $H(z)$ are FIR if and only if the bank of filters $\left\{P_{k}(z)\right\}$ and $\left\{Q_{k}(z)\right\}$ are FIR for all $k$. The structure from Fig. 5(b) is an $L$-channel, uniform, nonmaximally decimated filter bank. In our setting one side (analysis or synthesis) of this filterbank is usually known, and the task is to construct the other side so that the whole system has perfect reconstruction (PR) [3] property. For example, in the problem of signal reconstruction, $F(z)$ and thus $\left\{P_{k}(z)\right\}$ are known and the goal is to find the corresponding synthesis bank $\left\{Q_{k}(z)\right\}$. Recall that at the same time this is exactly the problem 


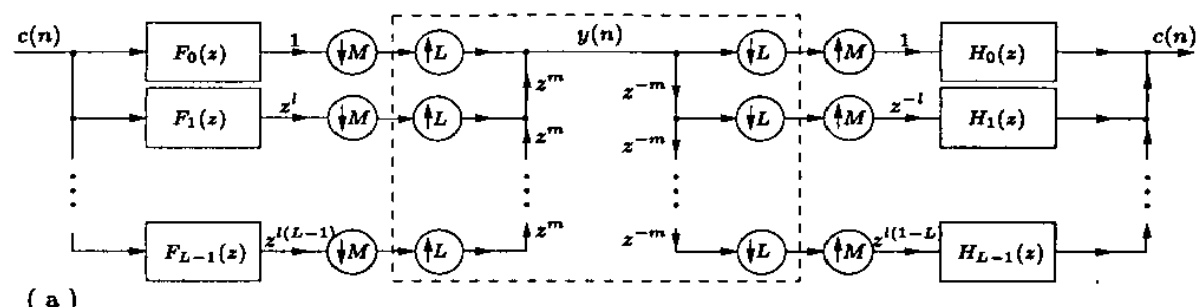

(a)

(b)

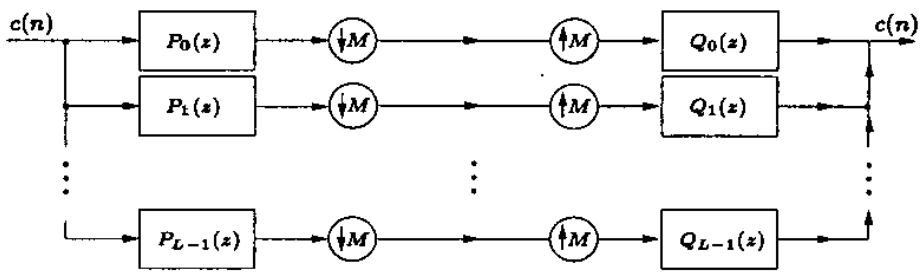

(c)

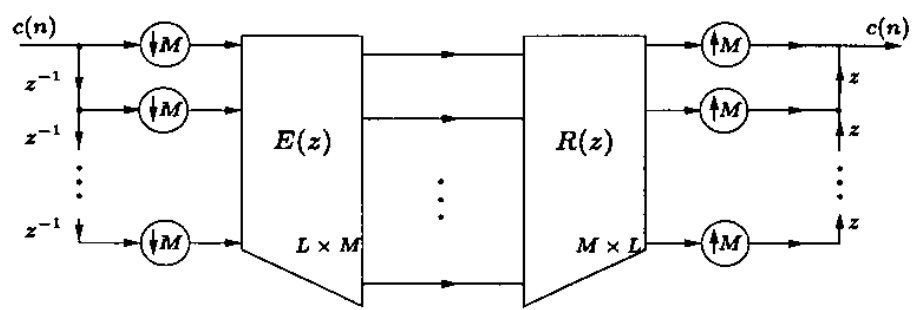

Fig. 5. (a)-(c) Further simplifications of fractional biorthogonal partners.

of constructing a RFBP $H(z)$, since it is uniquely defined by the filters $\left\{Q_{k}(z)\right\}$. The solution to these problems is well-known to the signal processing community. First, we define the $L \times M$ analysis and the $M \times L$ synthesis polyphase matrices $\mathbf{E}(z)$ and $\mathbf{R}(z)$ respectively

$$
\begin{gathered}
\mathbf{E}(z)=\left[\begin{array}{cccc}
E_{0,0}(z) & E_{0,1}(z) & \cdots & E_{0, M-1}(z) \\
E_{1,0}(z) & E_{1,1}(z) & \cdots & E_{1, M-1}(z) \\
\vdots & \vdots & \vdots & \vdots \\
E_{L-1,0}(z) & E_{L-1,1}(z) & \cdots & E_{L-1, M-1}(z)
\end{array}\right], \\
\mathbf{R}(z)=\left[\begin{array}{cccc}
R_{0,0}(z) & R_{0,1}(z) & \cdots & R_{0, L-1}(z) \\
R_{1,0}(z) & R_{1,1}(z) & \cdots & R_{1, L-1}(z) \\
\vdots & \vdots & \vdots & \vdots \\
R_{M-1,0}(z) & R_{M-1,1}(z) & \cdots & R_{M-1, L-1}(z)
\end{array}\right]
\end{gathered}
$$

with the Type-1 and Type- 2 polyphase components (of order $M$ this time) $E_{i, j}(z)$ and $R_{i, j}(z)$ defined by

$$
P_{k}(z)=\sum_{j=0}^{M-1} E_{k, j}\left(z^{M}\right) z^{-j}, \text { and } Q_{k}(z)=\sum_{i=0}^{M-1} R_{i, k}\left(z^{M}\right) z^{i}
$$

for $0 \leq k \leq L-1$. Now the system of Fig. 5(b) can be equivalently redrawn as in Fig. 5(c). We see that the problem of finding a RFBP of $F(z)$ becomes equivalent to that of finding a left inverse $\mathbf{R}(z)$ of an $L \times M$ matrix $\mathbf{E}(z)$. Obviously, when looking for a LFBP, we would find a right matrix inverse of $\mathbf{R}(z)$. Now it should be clear why we have included $L>M$ in our problem formulation. Based on these findings we prove the following theorem.
Theorem. Given the transfer function $F(z)$ and two co-prime integers $L$ and $M$, there exists a stable right fractional biorthogonal partner of $F(z)$ if and only if $L>M$, and the minimum rank of $\mathrm{E}\left(e^{j \omega}\right)$ pointwise in $\omega$ is $M$. For an FIR filter $F(z)$ there exists an FIR right fractional biorthogonal partner if and only if $L>M$, and the greatest common divisor (gcd) of all the $M \times M$ minors of $\mathbf{E}(z)$ is a delay. Here, the polyphase matrix $\mathbf{E}(z)$ is defined by (6)-(7). Analogous results hold for left FBPs as well.

Proof. We have shown that there exists a stable (FIR) RFBP of $F(z)$ if and only if there exists a stable (polynomial) left inverse of a (polynomial) matrix $\mathbf{E}(z)$. We know that fat matrices have no left inverse, so we immediately have $L>M$ as a necessary condition. Next, for the inverse of $\mathbf{E}\left(e^{j \omega}\right)$ to be stable, we need the full column rank of $\mathrm{E}\left(e^{j \omega}\right)$ pointwise in $\omega$, which is the same as saying that the minimum rank over all $\omega$ is $M$. Finally, from the linear systems theory we know that there is a left polynomial inverse of a $L \times M$ polynomial matrix if and only if the ged of all its $M \times M$ minors is a delay $[4,9]$.

$\nabla \nabla \nabla$

In the next section we consider one of the applications of this theory, namely the spline interpolation of discrete signals by using only FIR filters. Some other applications such as the equalization of communication channels are treated elsewhere [7].

\section{INTERPOLATION OF OVERSAMPLED SIGNALS}

Given a discrete time signal $x(n)$ and a function $\phi(t)$, we can almost always assume that $x(n)$ is obtained by sampling the continuous time signal $x(t)$ given by the model (1) at integers

$$
x(n)=\sum_{k=-\infty}^{\infty} c(k) \phi(n-k) .
$$


The only condition is that $\Phi\left(e^{j \omega}\right)$, the discrete time Fourier transform of $\phi(n)$ is nonzero for all $\omega$ [5]. This follows from the fact that in the Fourier domain we can write the above equation as $X\left(e^{j \omega}\right)=\Phi\left(e^{j \omega}\right) C\left(e^{j \omega}\right)$, and therefore can obtain the driving coefficients $c(n)$ via the inverse filtering $1 / \Phi\left(e^{j \omega}\right)$, if stable. This driving sequence can then be employed for signal reconstruction as in (1) or for the interpolation of discrete signals. The signal $x(n)$ interpolated by an integral factor $K$ is obtained by sampling $x(t)$ from (1) $K$ times more densely than at integers. Thus, the final stage of the interpolation process is shown in Fig. 6, with $\phi_{K}(t) \triangleq \phi(t / K)$. While in principle $\phi(t)$ can be chosen to be

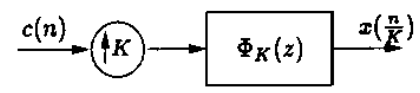

Fig. 6. The final stage of cubic spline interpolation; $\phi_{K}(n)$ is the cubic spline oversampled by $K$.
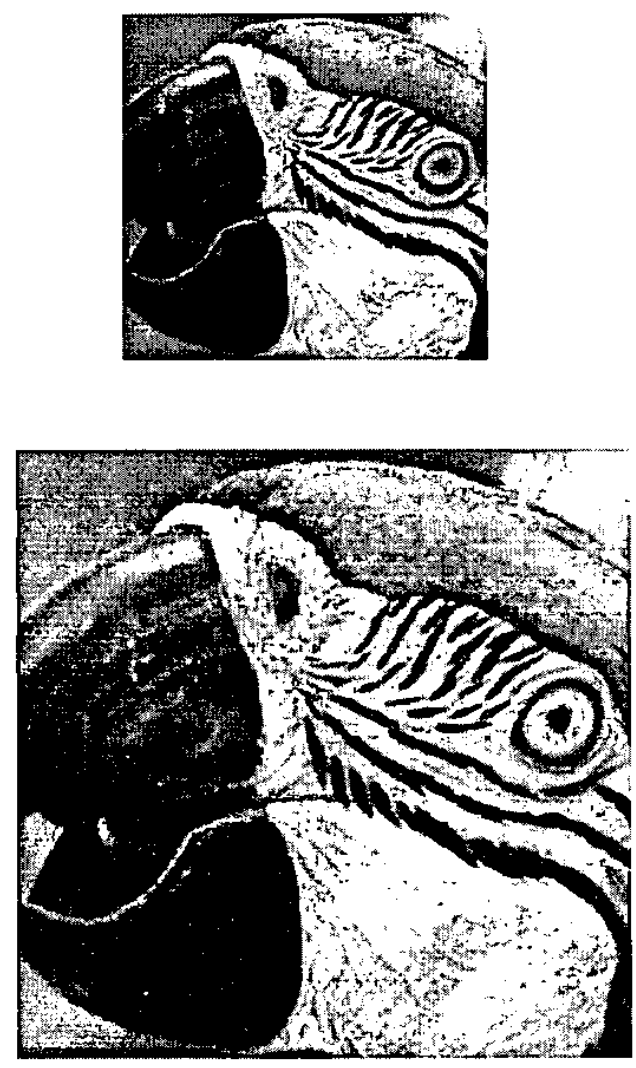

Fig. 7. FIR interpolation example: a region of the image oversampled by $6 / 5$ and its cubic spline interpolation with FIR filters.

just about any function, various researchers have traditionally used continuously differentiable interpolating functions such as cubic splines [1] to insure some smoothness properties of the resulting interpolant. The cubic spline and its oversampled version $\phi_{K}(t)$ for $K=3$ are shown in Fig. 1. Unfortunately, in the cubic spline case the inverse filtering $1 / \Phi\left(e^{j \omega}\right)$ is IIR and noncausal [2] and some recursive methods need to be used.

In the earlier work [5] it has been shown that cubic spline interpolation is achievable by FIR filtering if the original signal $x(t)$ is sampled at twice the integral rate to produce $x(n)$. However, following the discussion in Sec. 2.2 it is possible to perform the allFIR interpolation if $x(t)$ is oversampled by just a fraction $L / M$. By making $L=M+1$ and choosing $M$ large enough, this overhead can be made arbitrarily small.

In our example in Fig. 7 we used $L=6$ and $M=5$. The smaller image is a portion of the Parrots image, oversampled by $6 / 5$. In other words, this signal satisfies the model in Fig. 2(a). The driving sequence $c(n)$ was obtained as in Fig. 2(b) using the FIR filter $H(z)$. The interpolation as in Fig. 6 was then performed with $K=2$, and the result is shown in Fig. 7. Note that this procedure produces the exact cubic spline interpolant as opposed to another all-FIR method described in [6].

\section{CONCLUDING REMARKS}

Fractional biorthogonal partners (FBPs) represent a natural extension of the traditional biorthogonal partners defined for the integer upsampling and downsampling ratios. In this paper we have introduced the notion of FBPs and presented a way of their construction. The existence issues for FIR and for stable FBPs have also been treated. Lastly, we have provided one example where FBPs can occur, namely the all-FIR interpolation of slightly oversampled signals.

\section{REFERENCES}

[1] H. S. Hou and H. C. Andrews, "Cubic splines for image interpolation and digital filtering," IEEE Trans. Acoust., Speech. Signal Processing, vol. ASSP-26, pp. 508-517, 1978.

[2] M. Unser, A. Aldroubi, and M. Eden, "B-spline signal processing: Part I - Theory," IEEE Trans. Signal Processing, vol. 41, pp. 821-833, Feb. 1993.

[3] P. P. Vaidyanathan, Multirate Systems and Filter Banks. Prentice-Hall, Englewood Cliffs, NJ, 1995.

[4] P. P. Vaidyanathan and T. Chen, "Role of anticausal inverses in multirate filterbanks - Part I: System-theoretic fundamentals," IEEE Trans. Signal Proc., vol. 43(5), May 1995.

[5] P. P. Vaidyanathan and B. Vrcelj, "Biorthogonal partners and applications," IEEE Trans. Signal Processing, vol. 49(5), pp. 1013-1027, May 2001.

[6] B. Vrcelj and P. P. Vaidyanathan, "Efficient implementation of all-digital interpolation," IEEE Trans. Image Proc.

[7] B. Vrcelj and P. P. Vaidyanathan, "Fractional biorthogonal partners in fractionally spaced equalizers," submitted to ICASSP 2002.

[8] B. Vrcelj and P. P. Vaidyanathan, "Theory of MIMO biorthogonal partners and their application in channel equalization," Proceedings ICC, Helsinki, Finland, June 2001.

[9] X.-G. Xia, "New precoding for intersymbol interference cancellation using nonmaximally decimated multirate filterbanks with ideal FIR equalizers," IEEE Trans. Signal Processing, vol. 45(10), pp. 2431-2441, Oct. 1997. 